\title{
NJL model approach to diquarks and baryons in quark matter
}

\section{Blaschke}

Institute for Theoretical Physics, University of Wroclaw, 50-204; Wroclaw, Poland

Bogoliubov Laboratory for Theoretical Physics, Joint Institute for Nuclear Research, RU-141980 Dubna, Russia

E-mail: blaschkedift.uni.wroc.pl

\section{A. Dubinin*}

Institute for Theoretical Physics, University of Wroclaw, 50-204; Wroclaw, Poland

E-mail: aleksandr.dubinineift.uni.wroc.pl

\section{Zablocki}

Institute for Theoretical Physics, University of Wroclaw, 50-204; Wroclaw, Poland

E-mail: dan.zablocki@gmail.com

We describe baryons as quark-diquark bound states at finite temperature and density within the NJL model for chiral symmetry breaking and restoration in quark matter. Based on a generalized Beth-Uhlenbeck approach to mesons and diquarks we present in a first step the thermodynamics of quark-diquark matter which includes the Mott dissociation of diquarks at finite temperature. In a second step we solve the Bethe-Salpeter equation for the baryon as a quark-diquark bound state in quark-diquark matter. We obtain a stable, bound baryon even beyond the Mott temperature for diquark dissociation since the phase space occupation effect (Pauli blocking for quarks and Bose enhancement for diquarks) in the Bethe-Salpeter kernel for the nucleon approximately cancel so that the nucleon mass follows the in-medium behaviour of the quark and diquark masses towards chiral restoration. In this situation the baryon is obtained as a "borromean" three-quark state in medium because the two-particle state (diquark) is unbound while the three-particle state (baryon) is bound.

XXII International Baldin Seminar on High Energy Physics Problems,

15-20 September 2014

JINR, Dubna, Russia

\footnotetext{
*Speaker.
} 


\section{Thermodynamics of correlations in quark matter}

While numerous works have recently studied the thermodynamics of quark matter on the mean-field level including the effects of the medium dependence of the order parameters, not so much is known beyond the mean field, about hadronic correlations and their backreaction to the structure of the model QCD phase diagram and its thermodynamics. Here we will elaborate on the generalized Beth-Uhlenbeck form of the equation of state which is systematically extended from studying mesonic correlations [1] to the inclusion of diquark degrees of freedom [2,3]. To that end we will employ a Nambu-Jona-Lasinio-type quark model with fourpoint interactions in mesonic (quark-antiquark) and diquark (quark-quark) channels. We shall discuss here the importance of the interplay of the resonant states with the residual non-resonant ones in the continuum of scattering states. Due to the Levinson theorem both contributions have the tendency to compensate each other in quark matter above the Mott transition [4], see also [2, 3, 5, 6].

The most intriguing questions will occur when on the basis of this in-medium bosonized effective chiral quark model the next step of the hadronization program nucleon will be performed and diquarks will be "integrated out" in favor of baryons so that nuclear matter can be described in the model QCD phase diagram. A possible scheme for introducing baryons as quark-diquark bound states and integrating out the colored and therefore not asymptotically observable diquark fields has been suggested by Cahill and collaborators [7, 8, 9, 10]. It was afterwards elaborated by Reinhardt [11] and developed further by including the solitonic aspects of a field theoretic description of the nucleon [12]. However, the step to describe nucleonic many-body systems on this quark model basis has not been performed by these authors. Exploratory studies within the framework of an effective local NJL-type model for the quark-diquark interaction vertex have revealed a first glimpse at the modification of the nucleon spectral function in the different regions of the model QCD phase diagram, including chirally restored and color superconducting phases [13]. Again, the crucial step towards an equation of state for nuclear matter with this microscopic model for nucleons in the QCD phase diagram has not yet been done.

We will prepare the ground for a Beth-Uhlenbeck description of nuclear matter, to be discussed in future work. In particular, at zero temperature the structure of a Walecka model for nuclear matter shall emerge under specified conditions. Earlier work in this direction $[14,15,16]$ has demonstrated this possibility although no unified description of the nuclear-to-quark matter transition has been possible and the elucidation of physical mechanisms for the very transition between the hadronic and the quark matter phases of low energy QCD has been spared out. Or study aims at indicating directions for filling this gap by extending the discussion of the Mott mechanism for the dissociation of hadronic bound states of quarks within the NJL model description of low-energy QCD which has been given on the example ot two-particle correlations (mesons and diquarks) in [2] and sketching steps towards a Beth-Uhlenbeck description of nuclear matter where nucleons are treated as quarkdiquark correlations in quark matter [17].

\subsection{Quark matter thermodynamics in the NJL model}

We base the approach on the NJL model Lagrangian including vector and diquark interaction channels besides the standard scalar-pseudoscalar chirally symmetric interaction for the isospin symmetric case $\left(\mu_{u}=\mu_{d}=\mu\right.$ and $\left.m_{u}=m_{d}=m_{0}\right)$ 


$$
\begin{aligned}
\mathscr{L} & =\bar{q}\left[\mathrm{i} \partial-m_{0}+\gamma_{0} \mu\right] q+\mathscr{L}_{\text {int }}, \\
\mathscr{L}_{\text {int }} & =G_{\mathrm{S}}\left[(\bar{q} q)^{2}+\left(\bar{q} \mathrm{i} \gamma_{5} \tau q\right)^{2}\right]+G_{\mathrm{V}}\left(\bar{q} \mathrm{i} \gamma_{\mu} q\right)^{2}+G_{\mathrm{D}} \sum_{A=2,5,7}\left(\bar{q} \mathrm{i} \gamma_{5} \tau_{2} \lambda_{A} q^{C}\right)\left(\bar{q}^{C} \mathrm{i} \gamma_{5} \tau_{2} \lambda_{A} q\right) .
\end{aligned}
$$

Starting from the Lagrangian, we perform the usual bosonization by means of HubbardStratonovich transformations, thus integrating out the quark degrees of freedom to obtain a path integral representation of the partition function (and thus the thermodynamical potential $\Omega$ ) in terms of composite fields, mesons $\left(M=\sigma, \vec{\pi}, \omega_{\mu}\right)$ and (anti-)diquarks $\left(D, \bar{D}=\Delta_{A}, \Delta_{A}^{*}, A=2,5,7\right)$,

$$
\mathscr{Z}=\int \mathscr{D} \sigma \mathscr{D} \vec{\pi} \mathscr{D} \omega_{\mu} \mathscr{D} \Delta_{A} \mathscr{D} \Delta_{A}^{*} \mathrm{e}^{-\int \mathrm{d}^{4} x_{E}\left\{\frac{\sigma^{2}+\vec{\pi}^{2}}{4 G_{\mathrm{S}}}-\frac{\omega_{\mu}^{2}}{4 G_{\mathrm{V}}}+\frac{\left|\Delta_{A}\right|^{2}}{4 G_{\mathrm{D}}}\right\}+\frac{1}{2} \ln \operatorname{det}\left\{\beta S^{-1}\left[\sigma, \vec{\pi}, \omega_{\mu}, \Delta_{A}, \Delta_{A}^{*}\right]\right\}},
$$

where the inverse propagator is decomposed into mean field and fluctuation, $S^{-1}=S_{\mathrm{Q}}^{-1}-\Sigma$, with

$$
\begin{aligned}
S_{Q}^{-1} & =\left(\begin{array}{cc}
\left(\mathrm{i} z_{n}+\mu^{*}\right) \gamma_{0}-\gamma \cdot \mathbf{p}-m & \Delta_{\mathrm{MF}} \mathrm{i} \gamma_{5} \tau_{2} \lambda_{2} \\
\Delta_{\mathrm{MF}}^{*} \mathrm{i} \gamma_{5} \tau_{2} \lambda_{2} & \left(\mathrm{i} z_{n}-\mu^{*}\right) \gamma_{0}-\gamma \cdot \mathbf{p}-m
\end{array}\right), \\
\Sigma & =\left(\begin{array}{cc}
\sigma+\mathrm{i} \gamma_{5} \vec{\tau} \cdot \vec{\pi} & -\Delta_{A} \mathrm{i} \gamma_{5} \tau_{2} \lambda_{A} \\
-\Delta_{A}^{*} \mathrm{i} \gamma_{5} \tau_{2} \lambda_{A} & \sigma+\mathrm{i} \gamma_{5} \vec{\tau}^{T} \cdot \vec{\pi}
\end{array}\right) .
\end{aligned}
$$

Here, $z_{n}=(2 n+1) \pi T$ are the fermionic Matsubara frequencies and we have introduced the dynamical quark mass $m=m_{0}+\sigma$ and the effective chemical potential $\mu^{*}=\mu-\omega_{\mathrm{MF}}$; the vector meson has been redced to the meanfield value of its timelike component $\omega_{\mu}=\left(\omega_{\mathrm{MF}}, 0\right)$. In the present work, we consider NJL case, but we will restrict ourselves to the normal phase without color superconductivity $\left(\Delta_{M F}=0\right)$. Moreover, since we want to describe baryons as quark-diquark bound states, we have to go beyond Gaussian order in the diquark fields and include an infite set of diagrams which can be resummed to define the quark-diquark Bethe-Salpeter kernel, see [17] and references therein.

As a result, the thermodynamic potential $\Omega=-(T / V) \ln \mathscr{Z}$ takes the form

$$
\Omega_{\mathrm{tot}}=\Omega_{\mathrm{MF}}+\Omega_{\mathrm{M}}+\Omega_{\mathrm{D}}+\Omega_{\overline{\mathrm{D}}}+\Omega_{\mathrm{B}}+\Omega_{\mathrm{res}},
$$

where the meanfield part $\Omega_{\mathrm{MF}}=\Omega_{\text {cond }}+\Omega_{\mathrm{Q}}$ consists of a contribution from condensates

$$
\Omega_{\mathrm{cond}}=\frac{\sigma_{\mathrm{MF}}^{2}}{4 G_{\mathrm{S}}}-\frac{\omega_{\mathrm{MF}}^{2}}{4 G_{\mathrm{V}}}
$$

and from dynamical quarks

$$
\Omega_{\mathrm{Q}}=-2 N_{c} N_{f} \int \frac{d^{3} p}{(2 \pi)^{3}}\left\{E_{p}+T \ln \left[1+\mathrm{e}^{-\left(E_{p}-\mu^{*}\right) / T}\right]+T \ln \left[1+\mathrm{e}^{-\left(E_{p}+\mu^{*}\right) / T}\right]\right\} .
$$

The correlation contributions have the form

$$
\Omega_{\mathrm{X}}= \pm \frac{d_{X}}{2} \frac{T}{V} \operatorname{Tr} \ln \left(\beta^{c_{X}} S_{\mathrm{X}}^{-1}\right), \mathrm{X}=\mathrm{M}, \mathrm{D}, \overline{\mathrm{D}}, \mathrm{B},
$$


where for fermions ((B)aryons) holds the plus sign and $c_{X}=1$ while for bosons ((M)esons, (D)iquarks and their antiparticles $(\overline{\mathrm{D}}))$ holds the minus sign and $c_{X}=2$. The coeffficient $d_{X}$ in (1.8) is the degree of degeneracy of the state $X$ for which the inverse propagator can be given the form [2]

$$
S_{\mathrm{X}}^{-1}=\frac{1}{G_{\mathrm{X}}}-\Pi_{\mathrm{X}}\left(\mathrm{i} z_{n}, \mathbf{q}\right)
$$

with the effective coupling constant $G_{X}$ being defined by the coupling in the corresponding interaction channel of the NJL Lagrangian. The coupling for the baryon $G_{B}$ is discussed below. The polarization functions $\Pi_{\mathrm{X}}\left(\mathrm{i} z_{n}, \mathbf{q}\right)$ are defined in the RPA approximation as one-loop integrals which involve combinations of quark-quark, quark-antiquark and quark-diquark propagators with the corresponding vertex functions in the meson-, diquark-, and nucleon channels, respectively. Any residual terms which cannot be captured in the above approximation scheme are summarized in the residual thermodynamic potential $\Omega_{\text {res }}$. Here one finds in particular contributions beyond the Gaussian approximation in the meson fields as well as contributions to the baryon-baryon interaction. The required polarization loop integrals for mesons and diquarks are given in Ref. [2] for the NJL model. For the Polyakov loop extended NJL model one has to replace in the final expressions the Fermi- and Bose distribution functions for the color-carrying degrees of freedom $(\mathrm{Q}, \mathrm{D}, \overline{\mathrm{D}})$ by the generalized Fermi and Bose distribution functions, respectively [3, 17, 18].

\subsection{The baryon as quark-diquark state}

The quark-diqark polarization loop, which defines the baryon as an effective two-particle correlation in the medium, has in general a Dirac structure which, however, for a baryon at rest reduces to the scalar function (see also [17])

$$
\begin{aligned}
\Pi_{B}\left(\mathrm{i} z_{n}, \mathbf{0}\right)= & 4 m \int \frac{d^{3} p}{(2 \pi)^{3}} \frac{1}{4 E_{q} E_{D}}\left\{\frac{1-f^{-}\left(E_{q}\right)+g^{-}\left(E_{D}\right)}{\mathrm{i} z_{n}+E_{q}+E_{D}}-\frac{1-f^{+}\left(E_{q}\right)+g^{+}\left(E_{D}\right)}{\mathrm{i} z_{n}-E_{q}-E_{D}}\right. \\
& \left.-\frac{f^{+}\left(E_{q}\right)+g^{-}\left(E_{D}\right)}{\mathrm{i} z_{n}-E_{q}+E_{D}}+\frac{f^{-}\left(E_{q}\right)+g^{+}\left(E_{D}\right)}{\mathrm{i} z_{n}+E_{q}-E_{D}}\right\}
\end{aligned}
$$

where $E_{q}=\sqrt{p^{2}+m^{2}}$ and $E_{D}=\sqrt{p^{2}+m_{D}^{2}}$. For the PNJL model, the distribution functions for fermions $\left(f^{\mp}(E)=1 /[\exp (E \mp \mu) / T+1]\right)$, bosons $\left(g^{\mp}(E)=1 /[\exp (E \mp \mu) / T-1]\right)$ and their antiparticles need to be replaced by their generalizations for the PNJL case and get then strongly suppressed in the confining phase.

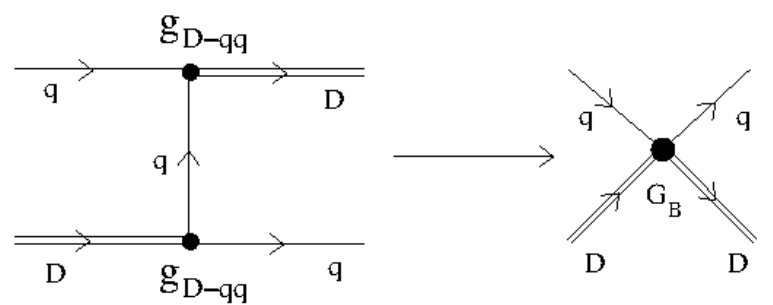

Figure 1: Diagrammatic representation of the instantaneous approximation to the quark-diquark interaction. 
In order to find the baryon mass, we make the instantaneous approximation for the quarkdiquark interaction vertex (see Fig. 1) which corresponds to identifying

$$
G_{B}=\frac{4 g_{D-q q}^{2}}{m}, g_{D-q q}^{2}=\frac{4 m_{D}}{\left.\frac{\partial \Pi_{D}(\omega, \mathbf{0})}{\partial \omega}\right|_{\omega=m_{D}}} \approx \mathrm{const},
$$

and solve the Bethe-Salpeter equation for the baryon as a bound quark-diquark state with a mass $m_{B}$ according to

$$
1-G_{B} \Pi_{B}\left(m_{B}, \mathbf{0}\right)=0 .
$$

Note that in contrast to Ref. [13] where $G_{B}=$ const was assumed, we follow here [17] and keep the dependence on the quark mass which stems from the instantaneous limit of the quark exchange kernel, see Fig. 1. Since with increasing temperature towards the chiral restoration the quark mass drops, this coupling gets enhanced and therefore the tendency for the nucleon being a "borromean state" which was already noted in [13] gets enhanced.

We can solve the mean field gap equation for the quark mass as a function of temperature and chemical potential for the NJL model, which will serve as inputs for solving the Bethe-Salpeter equations for mesons, diquarks and baryons in the medium. The masses of these states are obtained as poles of their propagators and are shown in Fig. 2. We observe that the chiral symmetry
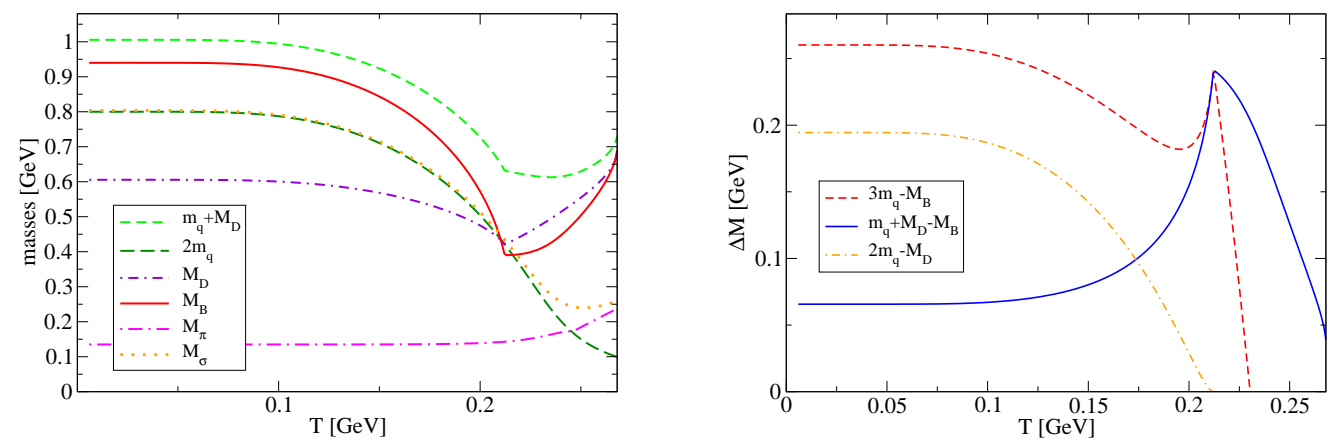

Figure 2: Left panel: Mass spectrum of pions, sigma mesons, diquarks and baryons as functions of the temperature $T$. Also shown are the relevant thresholds: $2 m_{q}$ for mesons and diquarks and $m_{q}+m_{D}$ for baryons. Right panel: Binding energy (mass defect) for baryons relative to the three-quark or the quark-diquark continuum, resp., and for diquarks relative to the two-quark continuum as a function of the temperature $T$. The baryon in hot quark matter is a "borromean" state: when the diquark becomes unbound, the baryon binding energy is still nonvanishing.

restoration which is reflected in the dropping quark mass function, induces a Mott effect for the pion and the scalar diquark. For both states the kernel of their Bethe-Salpeter equation contains a Pauli blocking term since they are composed of two fermions. This Pauli blocking partly compensates the effect of the quark selfenergies (dropping quark masses) and leads to a stabilization of the bound state masses against medium effects. This results in the crossing of the bound state masses with the continuum threshold, leading to the dissociation of these bound states. 
Interestingly, the situation is quite different for the baryons. Here, the bound state is composed of a fermion and a boson, so that there is a partial compensation of the phase space occupation effects in the Bethe- Salpeter kernel: the Pauli blocking for quarks gets partly compensated by a Bose enhancement for the diquarks. As a consequence of this, the baryon mass follows the behaviour of the quark-diquark continuum and does not experience a Mott effect in the range of temperatures we studied here, see Fig. 2. In the right panel of Fig. 2, we show the mass defect (binding energy) for diquarks and baryons, where for the latter we show two alternative definitions, i.e., relative to the mass of three quarks and relative to the sum of the quark and the diquark mass. As a remarkable fact we obtain that in a temperature region where the diquark became already unbound due to the Mott effect, the baryon is still bound. One can therefore say that the baryon in the vicinity of the chiral restoration transition behaves like a "borromean state": while the twoparticle state (diquark) got dissociated, the three-particle state (baryon) is still bound.

\subsection{Thermodynamics of meson, diquark and baryon correlations in quark matter}

The derivation of the diquark thermodynamic potential follows Ref. [2] where it was given for the NJL model and the form of a generalized Beth-Uhlenbeck EoS was derived. In such a formulation, the dissociation of bound states in a hot, dense medium by the Mott effect is given by the behaviour of the in-medium phase shifts which encode the analytic properties of the propagator in the complex energy plane in a polar representation. The analoguous result is given in the Ref. [3] for the PNJL model.

Now one is in the state to study the thermodynamics of the meson-, diquark-, and baryon correlations in a hot and dense medium. The equations of state of interest can be derived from the thermodynamical potential (1.5), e.g., for the pressure $P=-\Omega_{\text {tot }}$ or the energy density $\varepsilon=$ $\Omega_{\mathrm{tot}}-T \partial \Omega_{\mathrm{tot}} / \partial T-\mu \partial \Omega_{\mathrm{tot}} / \partial \mu$, whereby all corelation contributions have the form of a BethUhlenbeck equation

$$
\Omega_{\mathrm{X}}=\mp d_{\mathrm{X}} \int \frac{\mathrm{d}^{3} q}{(2 \pi)^{3}} \int_{0}^{\infty} \frac{\mathrm{d} \omega}{2 \pi}\left\{\omega \pm T \ln Y_{ \pm}^{-}(\omega) \pm T \ln Y_{ \pm}^{+}(\omega)\right\} \frac{d \delta_{\mathrm{X}}(\omega, \mathbf{q})}{d \omega}
$$

where the upper (lower) sign holds for fermions (bosons) and $Y_{ \pm}^{\mp}(\omega)=\left(1 \pm \mathrm{e}^{-\left(\omega \mp \mu_{\mathrm{X}}\right) / T}\right)$. The corresponding degeneracy factors are $d_{\pi}=d_{D}=d_{\vec{D}}=3$ and $d_{B}=4$. Note that in evaluating (1.7), (1.13) the zero-point energy terms will be dropped ("no sea" approximation).

For the phase shifts a general decomposition into a resonant (R) and a continuum (c) part can be made

$$
\delta_{\mathrm{X}}(\omega, \mathbf{q})=\delta_{\mathrm{X}, \mathrm{R}}(\omega, \mathbf{q})+\delta_{\mathrm{X}, \mathrm{c}}(\omega, \mathbf{q}),
$$

where both parts are uniquely defined by the propagator of the correlation, see [2]. The bound state mass is located at the jump of the phase shift from 0 to $\pi$ and this jump corresponds to a deltafunction in the Beth-Uhlenbeck formulas for the correlations. In the case when the continuum of the scattering states is separated by a sufficient energy gap from the bound state, it can be neglected and we obtain as a limiting case the thermodynamics of a statistical ensemble of on-shell correlations. We shall make use of this fact in the next section when deducing the Walecka model. 


\section{The Walecka model as a low temperature limit of the NJL model}

In this section, we show that the Walecka model in mean field approximation is contained in our model as limiting case. It is well known, that quarks undergo a Cooper instability leading to diquark condensates in this model due to the attractive quark-quark interaction. At zero temperature, this happens as $\mu^{*}>m$ or, if diquarks are bound, for $\mu^{*}>m_{D} / 2$. Here, we restrict our considerations to the region $\mu^{*}<\min \left\{m, m_{D} / 2\right\}$. However, by construction, the baryon appears as bound state, meaning $M_{\mathrm{B}} / 3<\min \left\{m, m_{D} / 2\right\}$. Consequently, there is a range in chemical potentials, where the appearance of baryons already affects the thermodynamics and leads to non-vanishing baryon densities,

$$
M_{\mathrm{B}} / 3<\mu^{*}<\min \left\{m, m_{D} / 2\right\},
$$

but quarks and diquarks are not excited yet. One could easily generalize this to finite temperatures, but then the effects of mesons should be taken into account.

\subsection{Deducing the equations}

Let us discuss this in more details. Our thermodynamic potential is given by

$$
\Omega_{\mathrm{tot}}=\Omega_{\mathrm{cond}}+\Omega_{\mathrm{Q}}+\Omega_{\mathrm{M}, \mathrm{R}}+\Omega_{\mathrm{M}, \mathrm{c}}+\Omega_{\mathrm{D}, \mathrm{R}}+\Omega_{\mathrm{D}, \mathrm{c}}+\Omega_{\overline{\mathrm{D}, \mathrm{R}}}+\Omega_{\overline{\mathrm{D}, \mathrm{c}}}+\Omega_{\mathrm{B}, \mathrm{R}}+\Omega_{\mathrm{B}, \mathrm{c}}
$$

with the explicit form of each term given above, and where we have decomposed the meson, diquark and baryon contributions in the respective resonance and continuum parts, as discussed above. At zero temperature, there are no contributions from the scattering states, due to the restriction $\mu^{*}<m$, which happen to be the thresholds defined in the imaginary parts of the meson and diquark polarization functions. The same holds for the scattering part of the baryons. This drastically simplifies the calculation as many terms drop out. However, the low temperature region is already of interest for the properties of neutron stars.

We choose the diquark coupling strength $G_{\mathrm{D}}$ small enough to avoid the diquark pole, and therefore avoid colored objects in this regime, the regime where hadrons are the only relevant degrees of freedom. Consequently there is no bound state contribution from that part either. Thus, (2.2) reduces to

$$
\Omega_{\mathrm{tot}}=\Omega_{\mathrm{cond}}+\Omega_{\mathrm{Q}}+\Omega_{\mathrm{M}, \mathrm{R}}+\Omega_{\mathrm{B}, \mathrm{R}} .
$$

For the quarks, only the vacuum part contributes in contrast to baryons, due to the condition $M_{\mathrm{B}} / 3<\mu^{*}$. Explicitly we have

$$
\Omega_{\mathrm{tot}}=\frac{\sigma^{2}}{4 G_{\mathrm{S}}}-\frac{\omega_{0}^{2}}{4 G_{\mathrm{V}}}-2 N_{c} N_{f} \int_{0}^{\Lambda} \frac{\mathrm{d}^{3} p}{(2 \pi)^{3}} E_{\mathbf{p}}-2 N_{f} \int_{0}^{\Lambda_{\mathrm{B}}} \frac{\mathrm{d}^{3} P}{(2 \pi)^{3}} E_{\mathrm{B}, \mathbf{P}}-\frac{N_{f}}{3 \pi^{2}} \int_{0}^{P_{F}} \mathrm{~d} P \frac{P^{4}}{\sqrt{M_{\mathrm{B}}^{2}+P^{2}}} .
$$

The integrals can be evaluated analytically, so that we can write

$$
\Omega_{\mathrm{tot}}=\frac{\sigma^{2}}{4 G_{\mathrm{S}}}-\frac{\omega_{0}^{2}}{4 G_{\mathrm{V}}}-\frac{1}{4 \pi^{2}}\left[3 F(\Lambda, m)+F\left(\Lambda_{\mathrm{B}}, M_{\mathrm{B}}\right)+G\left(P_{F}, M_{\mathrm{B}}\right)\right],
$$

with functions $F$ and $G$ given in the appendix. $P_{F}=\sqrt{\left(\mu_{\mathrm{B}}^{*}\right)^{2}-M_{\mathrm{B}}^{2}}$ is the Fermi momentum of the baryon, $\mu_{\mathrm{B}}^{*}=3 \mu^{*}$. 


\subsection{No sea approximation}

The transition to the original Walecka model needs a few more steps. First, we drop the vacuum contributions to the thermodynamic potential (2.5); this is what in the Walecka model is referred to as the no-sea approximation. The resulting thermodynamic potential reads

$$
\Omega_{\mathrm{tot}}=\frac{\sigma^{2}}{4 G_{\mathrm{S}}}-\frac{\omega_{0}^{2}}{4 G_{\mathrm{V}}}-\frac{1}{4 \pi^{2}} G\left(P_{F}, M_{\mathrm{B}}\right) .
$$

This already looks like a Walecka type model, but the scalar and vector fields here are connected to the quarks. We now need to clarify the connection between the quark order parameters and the corresponding baryonic ones used in the Walecka model. This is easily achieved by employing quark counting rules, where the vacuum contributions are absorbed into the vacuum nucleon mass: $M_{\mathrm{B}}=3\left(m_{0}+\sigma\right)-\kappa_{\mathrm{B}}=M_{\mathrm{B}, \mathrm{vac}}-3 \sigma_{\mathrm{med}}$. Motivated by our finding of Subsection 1.2 (see also Fig. 3), that the baryon mass defect (binding energy) in the model as a quark-diquark bound state is in lowest order medium independent, we adopt here in a first step a constant value of the parameter $\kappa_{\mathrm{B}}=261.7 \mathrm{MeV}$. The solution for the density dependent effective baryon mass is shown in Fig. 3 . The expression for $\sigma_{\text {med }}$ proportional to the scalar density $n_{\mathrm{S}}$ is then obtained from minimizing the

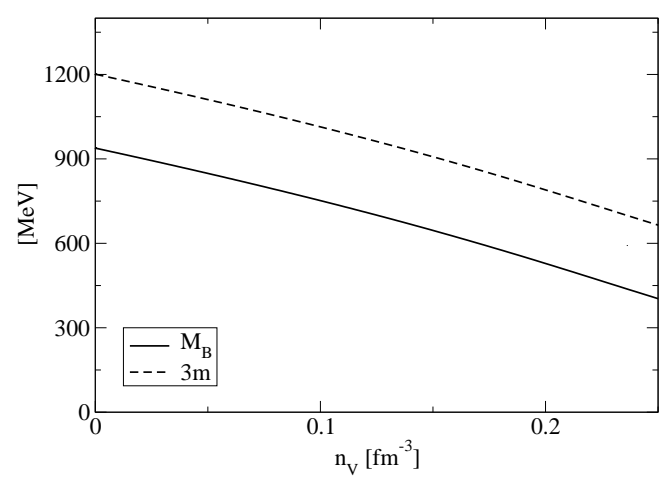

Figure 3: Baryon mass $M_{B}$ and three-quark continuum ( $\left.3 m\right)$ as function of the baryon density $n_{V}$.

thermodynamic potential (2.6) with respect to the order parameter $\sigma$ and setting $\Lambda=\Lambda_{\mathrm{B}}$,

$$
\begin{aligned}
\omega_{0} & =2 G_{\mathrm{V}} n_{\mathrm{V}} \\
n_{\mathrm{V}} & =\frac{2}{3 \pi^{2}} P_{F}^{3} \\
n_{\mathrm{S}} & =\frac{1}{4 \pi^{2}} F_{2}\left(P_{F}, M_{\mathrm{B}}\right)=\frac{M_{\mathrm{B}}}{\pi^{2}}\left[P_{F} E_{F}-M_{\mathrm{B}}^{2} \ln \frac{P_{F}+E_{F}}{M_{\mathrm{B}}}\right] \\
\sigma_{\text {med }} & =\frac{3 G_{\mathrm{S}}}{2 \pi^{2}} F_{2}\left(P_{F}, M_{\mathrm{B}}\right)=3\left(2 G_{\mathrm{S}}\right) n_{\mathrm{S}} .
\end{aligned}
$$

We can rewrite the pressure $P=-\Omega_{\text {tot }}$ and energy density $\varepsilon$ in terms of densities, keeping in mind 
that the baryon Fermi momentum is $P_{F}=\left(3 \pi^{2} n_{V} / 2\right)^{1 / 3}$,

$$
\begin{aligned}
P & =G_{\mathrm{V}} n_{\mathrm{V}}^{2}-9 G_{\mathrm{S}} n_{\mathrm{S}}^{2}+\frac{1}{4 \pi^{2}}\left(P_{F} E_{F}\left(\frac{2}{3} P_{F}^{2}-M_{\mathrm{B}}^{2}\right)+M_{\mathrm{B}}^{4} \ln \frac{P_{F}+E_{F}}{M_{\mathrm{B}}}\right) \\
\varepsilon & =-P+\mu n_{\mathrm{V}} \\
& =G_{\mathrm{V}} n_{\mathrm{V}}^{2}+9 G_{\mathrm{S}} n_{\mathrm{S}}^{2}+\frac{1}{4 \pi^{2}}\left(P_{F} E_{F}\left(2 P_{F}^{2}+M_{\mathrm{B}}^{2}\right)-M_{\mathrm{B}}^{4} \ln \frac{P_{F}+E_{F}}{M_{\mathrm{B}}}\right) .
\end{aligned}
$$

Note the factor 9, which appears in front of the scalar coupling strength. This reminds of the quark origin of this parameter and is in contrast to the vector coupling, where there is no prefactor, as the latter is entirely due to baryons. We use NJL parameters which would correspond to a constituent
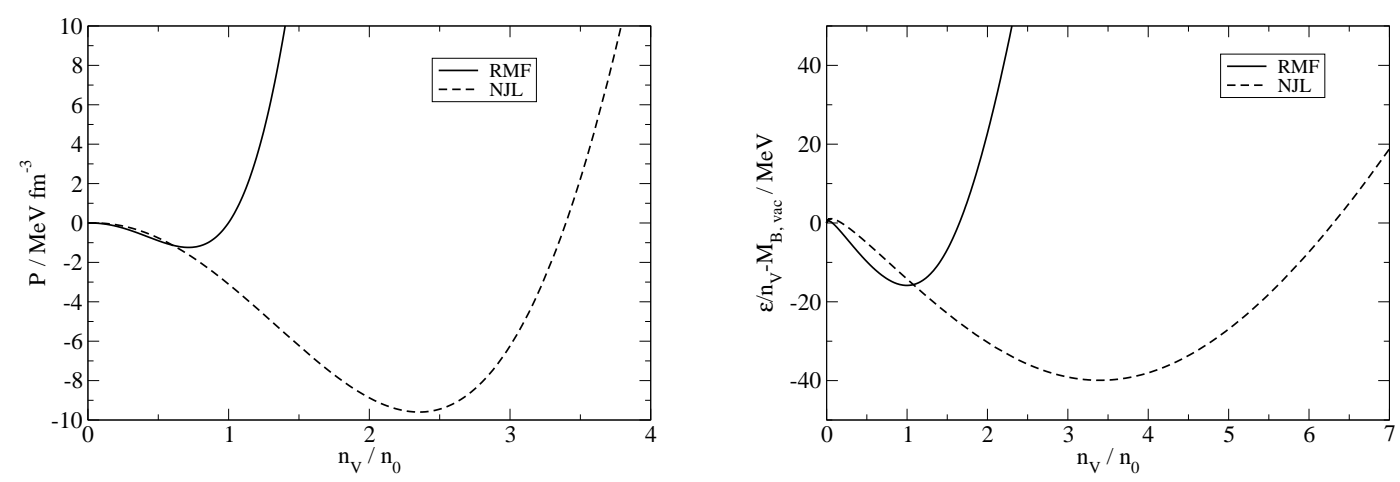

Figure 4: Comparison of the pressure and the binding energy per particle in a fitted Walecka model and an unmodified NJL parametrization $\left(G_{V}=4.5 G_{S}\right)$. For the empirical value of the saturation density we take $n_{0}=0.16 \mathrm{fm}^{-3}$.

quark mass of $m=400 \mathrm{MeV}$ [19], i.e., $m_{0}=5.588 \mathrm{MeV}, \Lambda=587.9 \mathrm{MeV}, G_{\mathrm{S}} \Lambda^{2}=2.442$ and $G_{\mathrm{V}}=0.5 G_{\mathrm{S}}$. Comparing this to the usual expressions for the Walecka model, we can define NJL model coupling strengths which would reproduce the Walecka model

$$
9 G_{\mathrm{S}, \text { Walecka }}=\frac{1}{2} \frac{g_{\sigma}^{2}}{m_{\sigma}^{2}}, G_{\mathrm{V}, \text { Walecka }}=\frac{1}{2} \frac{g_{\omega}^{2}}{m_{\omega}^{2}} .
$$

For values, reproducing the binding energy and saturation density [20]: $m_{\sigma}=550 \mathrm{MeV}, m_{\omega}=783$ $\mathrm{MeV}, g_{\sigma}=10.3$ and $g_{\omega}=12.7$, we can compare with the NJL model parametrization and find instead

$$
G_{\mathrm{S}}=0.33 G_{\mathrm{S}, \text { Walecka }}, G_{\mathrm{V}}=0.024 G_{\mathrm{V}, \text { Walecka }} .
$$

This leads already to saturating matter, even though at too high densities $\left(\approx 3.4 n_{0}\right)$ and with a too large binding energy $(\approx-40 \mathrm{MeV}$ instead of $-16 \mathrm{MeV})$, see Fig. 4 .

\section{Discussion and outlook}

The general aim of our work is the derivation of the thermodynamics for a meson-baryon system (quantum hadrodynamics $=$ QHD) on the basis of quark degrees of freedom and to explore 
the application limits of QHD, e.g., in the case of relativistic mean-field models for nuclear matter in heavy-ion collisions and neutron stars. The present contribution should be seen as a first step in this direction.

We could show that, when the analysis is restricted to a mean-field approximation for mesons and to small densities below the quark threshold $\left(\mu_{B}<3 m_{q}\right)$, the structure of the well-known $\sigma-\omega$ model (Walecka model) for nuclear matter emerges in the limit $T \rightarrow 0$. In this way we obtain from the NJL model an equation of state for nuclear matter at finite densities and temperatures, which has a saturation property. Taking the model as it is and using the standard model parameters, however, we could not reproduce the phenomenological values for the saturation properties. In fact, taking into account the simplicity of the model, this was not to be expected. Nevertheless, it should be viewed as an achievement of this work that a general path from a chiral quark model to the thermodynamics of nuclear matter could be identified.

In the present case, we find that the saturation density and the binding energy of the NJL nuclear matter, which result from the interplay between the scalar and vector mean fields, are too large. In order to obtain the phenomenological values, one should take in to account additional effects which lead to an enhancement of both, the repulsion (vector field) and of the attraction (scalar field). Here we think that the consideration of higher-order terms in the meson fields could be a promising option, because a consistent bosonization of the NJL model requires the consideration and renormalization of terms up to the fourth order, which would otherwise lead to divergencies. In this way, one obtains, for example, from the NJL model the successful hadronic linear $\sigma$ model, see [21] and references therein. Alternatively, terms of higher order can already be introduced on the level of the original quark Lagrangian, leading, however, to new coupling constants (see [22] and refs. therein). This freedom has been used, e.g., by Huguet et al. [23] to successfully reproduce the saturation properties of nuclear matter at $T=0$ in this way. Other interesting approaches to describe nuclear matter based on quark degrees of freedom are discussed in [2]. Besides this, we have omitted terms in $\Omega_{\text {res }}$ which correpond to quark exchange terms between baryons and would result in a repulsive Pauli blocking contribution at high densities. Considering such higher order terms one should eventually be able to reduce the binding energy and saturation density towards their physical values!

Finally, we want to point out that a suitable confining mechanism has to be present in a quarkmatter model, in order to exclude the appearance of unphysical degrees of freedom already in the experimentally well studied nuclear-matter regime. In this context the Polyakov loop has proven to be an interesting tool, although it does not involve all aspects of confinement. This is in particular the case at low temperatures and for $T=0$.

\section{Acknowledgement}

We are grateful to M. Buballa for his continued interest in the progress of this work and his critical remarks which helped improving this manuscript. This work was supported by the Polish National Science Centre (NCN) under contract number UMO-2011/02/A/ST2/00306 (A.D., D.B., D.Z.) and by the German Deutsche Forschungsgemeinschaft (DFG) under contract number BU 2406/1-1 (D.Z.). A.D. acknowledges a grant from the Institute for Theoretical Physics of the 
University of Wroclaw under contract No. 1356/M/IFT/13 and support by the Bogoliubov-Infeld programme for scientific collaboration between Polish Institutions and the JINR Dubna.

\section{A. Basic integrals}

We list some integrals encountered in the zero temperature evaluation of quark loop integrals

$$
\begin{aligned}
& F(x, y)=8 \int^{x} \mathrm{~d} p p^{2} \sqrt{p^{2}+y^{2}}=\left(x \sqrt{x^{2}+y^{2}}\left(2 x^{2}+y^{2}\right)-y^{4} \ln \frac{x+\sqrt{x^{2}+y^{2}}}{y}\right) \\
& G(x, y)=\frac{8}{3} \int^{x} \mathrm{~d} p \frac{p^{4}}{\sqrt{p^{2}+y^{2}}}=\left(x \sqrt{x^{2}+y^{2}}\left(\frac{2}{3} x^{2}-y^{2}\right)+y^{4} \ln \frac{x+\sqrt{x^{2}+y^{2}}}{y}\right)
\end{aligned}
$$

Partial derivatives of these functions are encountered in the evaluation of generalized gap equations; here we require

$$
F_{2}(x, y) \equiv \frac{\partial F(x, y)}{\partial y}=4 y\left[x \sqrt{x^{2}+y^{2}}-y^{2} \ln \frac{x+\sqrt{x^{2}+y^{2}}}{y}\right] .
$$

\section{References}

[1] J. Hüfner, S. P. Klevansky, P. Zhuang and H. Voss, Annals Phys. 234, 225 (1994).

[2] D. Blaschke, M. Buballa, A. Dubinin, G. Röpke and D. Zablocki, Annals Phys. 348, 228 (2014).

[3] D. Blaschke, A. Dubinin and M. Buballa, arXiv:1412.1040 [hep-ph].

[4] A. Wergieluk, D. Blaschke, Y. L. Kalinovsky and A. Friesen, Phys. Part. Nucl. Lett. 10, 660 (2013).

[5] K. Yamazaki and T. Matsui, Nucl. Phys. A 913, 19 (2013).

[6] S. Roessner, T. Hell, C. Ratti and W. Weise, Nucl. Phys. A 814, 118 (2008).

[7] R. T. Cahill, Austral. J. Phys. 42, 171 (1989).

[8] R. T. Cahill, J. Praschifka, C. Burden, Austral. J. Phys. 42, 161 (1989).

[9] C. J. Burden, R. T. Cahill and J. Praschifka, Austral. J. Phys. 42, 147 (1989).

[10] J. Praschifka, C. D. Roberts and R. T. Cahill, Phys. Rev. D36, 209 (1987).

[11] H. Reinhardt, Phys. Lett. B244, 316 (1990).

[12] U. Zückert, R. Alkofer, H. Weigel and H. Reinhardt, Phys. Rev. C 55, 2030 (1997).

[13] J. -C. Wang, Q. Wang and D. H. Rischke, Phys. Lett. B704, 347 (2011).

[14] W. Bentz and A. W. Thomas, Nucl. Phys. A696, 138 (2001).

[15] W. Bentz, T. Horikawa, N. Ishii and A. W. Thomas, Nucl. Phys. A720, 95 (2003).

[16] S. Lawley, W. Bentz and A. W. Thomas, J. Phys. G G32, 667 (2006).

[17] E. Blanquier, J. Phys. G 38, 105003 (2011).

[18] H. Hansen, W. M. Alberico, A. Beraudo, A. Molinari, M. Nardi and C. Ratti, Phys. Rev. D 75, 065004 (2007). 
[19] H. Grigorian, Phys. Part. Nucl. Lett. 4, 223 (2007).

[20] M. Buballa, Nucl. Phys. A 611, 393 (1996).

[21] S. P. Klevansky, Rev. Mod. Phys. 64, 649 (1992).

[22] S. Benic, Eur. Phys. J. A 50, 111 (2014).

[23] R. Huguet, J. C. Caillon and J. Labarsouque, Nucl. Phys. A 809, 189 (2008). 